\title{
Pulsing blue light through closed eyelids: effects on acute melatonin suppression and phase shifting of dim light melatonin onset
}

This article was published in the following Dove Press journal:

Nature and Science of Sleep

2 December 2014

Number of times this article has been viewed

\section{Mariana G Figueiro \\ Barbara Plitnick \\ Mark S Rea}

Lighting Research Center, Rensselaer Polytechnic Institute, Troy, NY, USA

Correspondence: Mariana G Figueiro Lighting Research Center, Rensselaer Polytechnic Institute, 2I Union Street,

Troy, NY 12180 , USA

$\mathrm{Tel}+\mathrm{I} 5186877100$

$\mathrm{Fax}+\mathrm{I} 5186877120$

Email figuem@rpi.edu
Abstract: Circadian rhythm disturbances parallel the increased prevalence of sleep disorders in older adults. Light therapies that specifically target regulation of the circadian system in principle could be used to treat sleep disorders in this population. Current recommendations for light treatment require the patients to sit in front of a bright light box for at least 1 hour daily, perhaps limiting their willingness to comply. Light applied through closed eyelids during sleep might not only be efficacious for changing circadian phase but also lead to better compliance because patients would receive light treatment while sleeping. Reported here are the results of two studies investigating the impact of a train of $480 \mathrm{~nm}$ (blue) light pulses presented to the retina through closed eyelids on melatonin suppression (laboratory study) and on delaying circadian phase (field study). Both studies employed a sleep mask that provided narrowband blue light pulses of 2 -second duration every 30 seconds from arrays of light-emitting diodes. The results of the laboratory study demonstrated that the blue light pulses significantly suppressed melatonin by an amount similar to that previously shown in the same protocol at half the frequency (ie, one 2-second pulse every minute for 1 hour). The results of the field study demonstrated that blue light pulses given early in the sleep episode significantly delayed circadian phase in older adults; these results are the first to demonstrate the efficacy and practicality of light treatment by a sleep mask aimed at adjusting circadian phase in a home setting.

Keywords: circadian phase, dim light melatonin onset, light through closed eyelids, blue light, sleep

\section{Background}

Impaired sleep is a common problem among older adults and is associated with reduced daytime function, increased health care costs, increases in falls and injuries, and reduced quality of life. ${ }^{1-4}$ Studies suggest that $40 \%-70 \%$ of older adults suffer from chronic sleep disturbances; fewer than $30 \%$ report having no sleep disturbances. ${ }^{5,6}$ Poor sleep is a major risk factor for developing mood disorders $;{ }^{7,8} 20 \%$ of individuals with insomnia also have depression. ${ }^{9}$ Conversely, mood disorders may cause disturbed sleep in older adults. Other common causes of disturbed sleep among older adults include chronic medical conditions, prescribed medication, and nonprescription drugs. In all of these situations, circadian rhythm disturbances play a role. Circadian rhythm disturbances parallel the increased prevalence of sleep disorders with increased age and cognitive impairment. ${ }^{10}$ Therefore, therapies, especially non-pharmacological treatments, specifically targeting regulation of the circadian system could be used to alleviate many sleep disorders in older adults.

While the majority of studies to date investigated the effect of light on sleep and behavior in Alzheimer's disease and related dementia patients, a few studies 
have examined the use of light to treat sleep disorders in older adults without symptoms of dementia. Kirisoglu and Guilleminault ${ }^{11}$ investigated how light treatment affected insomnia in older adults. Thirty participants with sleep complaints or disruptions were given 60 days of light treatment from a commercially available light box of an illuminance of 10,000 lux at eye level, for either a short exposure of 20 minutes or long exposure of 45 minutes. Those who received the longer light exposure consistently showed better sleep than the short-exposure group, both during the experiment and at the follow-up point. Data showed that total sleep time improved by 91 minutes in the long-exposure group and by 14 minutes in the short-exposure group. The authors concluded that light can be used to treat insomnia and that longer exposures were more effective.

Münch et $\mathrm{al}^{12}$ evaluated whether evening light treatment could delay circadian rhythms, reduce daytime sleepiness, and improve the overall sleep quality of older adults. After a baseline period to assess each participant's circadian phase, ten participants received 2 hours of light applied 3 hours prior to bedtime on days 5-8 of the study, followed by another circadian phase assessment. The light treatment was provided by a light box (either polychromatic white fluorescent light at 4,100 K, delivering 1,313 lux at eye level, or "blue-enriched" polychromatic white fluorescent light, delivering 654 lux at eye level). No differences were found between the groups in sleep time, amount of sleep, or sleep quality. However, participants reported an increase in alertness during light treatment, with no change in alertness before and after treatment.

Lieverse et $\mathrm{al}^{13}$ investigated the effect of bright light treatment in older adults suffering from nonseasonal major depressive disorder. They recruited 89 patients aged 60 years and older for a double-blind, placebo-controlled, randomized clinical trial. During the 3-week light treatment, participants $(\mathrm{n}=40)$ received 1 hour of 7,500 lux at eye level of pale-blue filtered light (active light) in the morning, delivered via light boxes. The control condition $(n=44)$ used a red filter instead of the pale-blue filter. Results showed a reduction in depressive symptoms for those receiving the active light, as measured by a change in the Hamilton Rating Scale for Depression $^{14}$ from baseline to intervention. Reduction in depressive symptoms was observed 3 weeks after treatment withdrawal, suggesting a strong after-effect of the treatment. Light treatment also improved sleep efficiency in this population.

While light has shown promise to help improve sleep in older adults suffering from insomnia or depression, compliance may be a barrier to the successful application of light treatment. ${ }^{15}$ Current recommendations for light treatment require the patients to sit in front of a bright light box for at least 1 hour daily, perhaps limiting their willingness to comply. Moreover, light levels tend to be very high and glare may be an issue, especially with the older population who tend to be more sensitive to glare. One way to increase compliance could be to deliver light through closed eyelids during sleep. As previously shown under laboratory conditions, people can, in fact, continue to sleep during retinal light exposures and not even be aware of these light exposures. ${ }^{16}$ The effectiveness of light exposures for adjusting circadian phase can also be greatest when people are asleep. People are usually asleep when core body temperature is at its minimum $\left(\mathrm{CBT}_{\min }\right)$, approximately 2-3 hours prior to natural waking. Retinal light exposure just prior to $\mathrm{CBT}_{\min }$ will maximally delay the timing of the biological clock, while light exposure just after $\mathrm{CBT}_{\min }$ will maximally advance circadian phase. Therefore, a carefully timed light exposure during sleep could not only be the most efficacious at changing circadian phase but also would likely be less objectionable to patients because they would have little or no conscious memory of the light exposure.

Using the model of the spectral transmittance of the human eyelid proposed by Bierman et al, ${ }^{17}$ together with the model of human circadian phototransduction by Rea et al, ${ }^{18,19}$ Figueiro and $\mathrm{Rea}^{16}$ developed and tested a sleep mask that could deliver $527 \mathrm{~nm}$ (green) light from light-emitting diodes (LEDs) through closed eyelids. In their study, the sleep mask delivered between 17,000 lux and 75,000 lux (33-131 W/m²) of green light to the subjects' eyelids; the amount of light depended upon the individual's measured eyelid spectral transmittance. Results showed that the continuous green light significantly delayed dim light melatonin onset (DLMO) and acutely suppressed nocturnal melatonin. Logical extensions from the model of human circadian phototransduction by Rea et al ${ }^{18,19}$ suggested that pulsing a $480 \mathrm{~nm}$ (blue) light should also be effective for stimulating the human circadian system, thereby significantly reducing the heat generated by the LEDs in the sleep mask. In fact, the efficacy of a sleep mask delivering 2-second pulses of blue light every 1 minute for 1 hour was shown to significantly suppress melatonin and phase delay DLMO in a population of young-to-middle-aged adults in a laboratory setting. ${ }^{20}$

Reported here are the results of two studies investigating the impact of a train of narrowband blue light pulses presented to the retina through closed eyelids on suppressing melatonin (laboratory study) and on delaying circadian 
phase (field study). Both studies employed a sleep mask that provided a train of light pulses of 2-second duration every 30 seconds from arrays of LEDs having a peak spectral emission at $480 \mathrm{~nm}$. The general goals of these studies were to confirm and extend previous results using the sleep mask. Specifically the main goal of the laboratory study was to determine whether a frequency of blue light pulses higher than that used in the previous study ${ }^{20}$ would be more effective at suppressing nocturnal melatonin; the goal of the field study was to determine whether it was possible to translate previous laboratory findings on light-induced phase shifting with young subjects to an older population in their home environments.

\section{Methods: laboratory study Participant selection}

Eleven participants (seven females) mean age \pm standard deviation (SD) $37.6 \pm 9$ years came to the laboratory on two consecutive Friday nights. Subjects were recruited to participate in the study from an electronic posting at Rensselaer Polytechnic Institute (RPI) in Troy, NY. All subjects were screened for major health problems and reported not taking any pharmaceuticals or medications. All subjects were asked to maintain a regular sleep/wake schedule (bedtimes by $11 \mathrm{pm}$ and wake times by $7 \mathrm{am}$ ) during the 2-week experiment. Participants completed their sleep/wake diaries for 3 weeks beginning 1 week prior to the start of the experiment. These diaries documented bedtimes, wake times, caffeine consumption, and quality of sleep. Because all subjects were employed, they had fixed schedules during the work week, which made compliance to the imposed schedule easier. An experimenter verified that all subjects complied with the imposed sleep/ wake schedule by checking their sleep logs. All subjects were nonsmokers and reported not suffering from any sleep disorders. All subjects provided written informed consent approved by RPI's Institutional Review Board and were paid for their participation. Subjects were asked to refrain from alcohol and caffeine starting 12 hours prior to coming to the laboratory both Friday nights. The study was conducted in accordance with the Declaration of Helsink $\mathrm{i}^{21}$ and conformed to international ethical standards.

Every subject completed a Munich Chronotype Questionnaire $^{22}$ prior to the study. This questionnaire is used to rate subjects" "morningness" or "eveningness". In brief, the subjects were asked to rate themselves as extreme early type (0), moderate early type (1), slight early type (2), normal type (3), slight late type (4), moderate late type (5), and extreme late type (6). Average $\pm \mathrm{SD}$ chronotype was
$2.5 \pm 1.5$, midway between a "slightly early" score of 2.0 and a "normal" score of 3.0 .

\section{Lighting conditions}

Light was delivered to both retinae through closed eyelids using the sleep mask previously described by Figueiro et al. ${ }^{20}$ Every sleep mask contained two blue LED arrays (wavelength of peak intensity $\left[\lambda_{\text {max }}\right]=480 \mathrm{~nm}$, full-width-half-maximum $=$ $24 \mathrm{~nm}$ ), one for each eyelid. When the sleep mask's elastic strap was placed around the back of the head, the LED arrays were positioned in front of the eyelids. The light-stimulus condition was a train of blue light pulses: 120 pulses of 2 -second duration presented every 30 seconds for 1 hour.

The prescribed corneal irradiance levels were based upon circadian stimulus $(\mathrm{CS})^{23}$ calculations. CS reflects the spectral and absolute sensitivities of the human circadian system and is defined in terms of equivalence to the estimated percentage, from $0 \%$ to $70 \%$, of light-induced nocturnal melatonin suppression following 1-hour exposure to the retina (eyes open) from a non-pulsing light through a fixed pupil of $2.3 \mathrm{~mm}$ diameter. Since the eyelids were closed for the present study, it was necessary to estimate eyelid transmission at $480 \mathrm{~nm}$. The mean eyelid spectral transmittance function from Bierman et $\mathrm{al}^{17}$ was used to determine corneal irradiance, and thus CS, for a closed eyelid. Because individual eyelid transmittance measurements were not made for the subjects in the study, and to ensure that all subjects received a significant stimulus, the irradiance for the $480 \mathrm{~nm}$ blue stimulus was set to provide a CS of 0.30 for the thickest eyelids measured in Bierman et al. ${ }^{17}$ Based upon a mean eyelid transmittance at $480 \mathrm{~nm}$ of $0.28 \%$, the mean $\pm \mathrm{SD}$ eyelid irradiances from the flashing blue light mask needed to achieve this CS value were $225 \mathrm{~W} /$ $\mathrm{m}^{2} \pm 7.6 \mathrm{~W} / \mathrm{m}^{2}$ or 27,493 lux \pm 966 lux. The corresponding CS for a person having mean eyelid transmittance was 0.43 . Note that the retinal light exposure (irradiance $\times$ time) in the present experiment is only $7 \%$ of that from a continuous, 1 -hour exposure. The uncertainties associated with quantifying retinal light exposures are described by Figueiro et al. ${ }^{20}$

\section{Procedures}

All subjects arrived at the laboratory at $10 \mathrm{pm}$ and were immediately placed in a dim room illuminated with red LEDs $\left(\lambda_{\max }=\right.$ $640 \mathrm{~nm}$ ) delivering $<2$ lux at the cornea. At $11 \mathrm{pm}$, each subject was asked to lie down on a mattress on the floor and to wear the sleep mask. As soon as the subjects were comfortable, a registered nurse or a phlebotomist started collecting the first blood sample from every subject using a small butterfly 23-gauge needle. Soon after the first blood samples were collected in dim 
light, the sleep masks were, as appropriate, energized. (Counterbalancing across subjects, participants experienced a treatment night where the sleep mask was energized, and a dark control night, where the sleep mask was worn, but not energized.) During the 1 hour when subjects were wearing the sleep mask, they were required to remain on the mattresses and allowed to sleep. At midnight, a second blood sample was obtained from the subjects, after which the sleep mask was removed and subjects were allowed to go home. The first blood sample was collected in dim light (T1) during both nights (control and treatment) and the second blood sample was collected following 1 hour (T2) of darkness (control night) or after exposure to the train of blue light pulses (treatment night).

Blood samples were centrifuged and frozen at $-20^{\circ} \mathrm{C}$ until assayed for melatonin concentrations by radioimmunoassay using a commercially available kit from Labor Diagnostika Nord (Nordhorn, Germany). The limit of detection was $2.3 \mathrm{pg} / \mathrm{mL}$ and the intra-assay and inter-assay coefficients of variability were determined to be $9.7 \%$ and $12.1 \%$, respectively.

\section{Methods: field study Participant selection}

Ten subjects (six females) mean age \pm SD $70 \pm 3.7$ years completed a 2-week study. Subjects were recruited to participate in the study from an electronic posting at RPI. All subjects were screened for major health problems. Prior to accepting them into the study, a research nurse screened potential subjects for sleep apnea, restless leg syndrome, capability of signing the consent form, macular degeneration, and cognitive impairment. Eight participants reported going to bed by $11 \mathrm{pm}$ and two participants reported going to bed by $12.30 \mathrm{am}$. All subjects provided written informed consent approved by RPI's Institutional Review Board and were paid for their participation. The study was conducted in accordance with the Declaration of Helsinki ${ }^{21}$ and conformed to international ethical standards.

\section{Lighting conditions}

The same sleep mask used in the laboratory study was used in this field study.

\section{Procedures}

During the baseline week, participants led their normal lives. Over the 2-week study, participants were asked to wear the Daysimeter $^{24,25}$ while awake and an actigraph ${ }^{26}$ at all times, except for when showering. At the end of the baseline week, participants collected saliva samples for phase assessment by DLMO. Saliva collection times were between $7 \mathrm{pm}$ and $11 \mathrm{pm}$ for eight subjects and between $6 \mathrm{pm}$ and $10 \mathrm{pm}$ for two subjects. Participants collected saliva samples at home (timing was based upon their sleep schedule), every 30 minutes during a 4.5-hour window that terminated approximately 1.5 hours after the predicted DLMO calculated using their habitual sleep schedules. Participants were instructed to remain in dim light and refrain from eating and drinking during the saliva collection period. Saliva samples were refrigerated until the next morning, when the research nurse collected the samples and brought them to the laboratory, where they were immediately frozen.

During the intervention week, which started the following day, the sleep mask was programmed to start the train of blue light pulses 1 hour after bedtimes and continue for 2 hours, ending no later than 1 hour prior to a subject's predicted $\mathrm{CBT}_{\min }$. The subjects' predicted $\mathrm{CBT}_{\text {min }}$ values were determined using their sleep log times ${ }^{27}$ obtained prior to the experiment; the range of $\mathrm{CBT}_{\text {min }}$ values for the ten subjects was between $3 \mathrm{am}$ and $4.30 \mathrm{am}$. Therefore, the train of blue light pulses always occurred during the expected delay portion of the phase response curve. At the end of the intervention week, participants again collected and refrigerated saliva samples. As for the baseline week, the samples were subsequently collected and frozen by the research nurse.

Melatonin concentrations in the saliva samples were determined by radioimmunoassay using a commercially available kit from Labor Diagnostika Nord (Nordhorn, Germany). The limit of detection was $1.4 \mathrm{pg} / \mathrm{mL}$ and the intra-assay and inter-assay coefficients of variability were determined to be $11.4 \%$ and $14.6 \%$, respectively. All saliva samples from both collection periods were assayed in the same batch.

During the intervention week, participants were asked to rate their sleepiness levels four times a day (waking, noon, dinner, and bedtime) using the Karolinska Sleepiness Scale (KSS), ${ }^{28}$ a standardized self-assessment of subjective sleepiness. The KSS ranges from 1 to 9, where 1= "very alert", $3=$ "rather alert", 5= "neither alert nor sleepy", 7= "sleepy, but no difficulty remaining awake", and 9= "very sleepy, fighting sleep, an effort to remain awake". To help with compliance, subjects did not have to write down the exact times of their KSS assessments. Rather, they simply indicated wake, lunch, dinner, or bedtime. Consequently, every subject filled out the questionnaire at slightly different clock times.

\section{Phase shifting}

DLMO times were calculated using one of two techniques published in the literature, either by taking the average of the three lowest points plus twice the SD of these points (" $3 \mathrm{~L}$ ") ${ }^{29}$ or by taking the average of the five continuous lowest points 
plus $15 \%$ of the five continuous highest points (“5H/5L"). ${ }^{30} \mathrm{The}$ different techniques were employed to account for individual differences in the observed melatonin profiles and to account for the fact that four subjects did not provide enough saliva for melatonin assay at the latter part of the night..$^{31}$ The $3 \mathrm{~L}$ method was used for those subjects with steep melatonin profiles whereas the $5 \mathrm{H} / 5 \mathrm{~L}$ method was used for those with shallow melatonin profiles. The $5 \mathrm{H} / 5 \mathrm{~L}$ method better characterized DLMO than the $3 \mathrm{~L}$ method for shallow profiles because the latter method is based upon the first three, lowest melatonin concentrations, which were very often the same value with no $\mathrm{SD}$. The $5 \mathrm{H} / 5 \mathrm{~L}$ method was used to calculate DLMO for four subjects and the $3 \mathrm{~L}$ method was used to calculate DLMO for six subjects. For every subject, the same threshold method was used in weeks 1 and 2. Changes in circadian phase were calculated as the difference between DLMO at baseline and DLMO after the light intervention week. In order to determine whether baseline DLMOs and intervention DLMOs were significantly different, two-tailed paired Student's $t$-tests were performed. All statistical analyses were performed using PASW Statistics 18.0 Software (SPSS, Chicago, IL, USA).

\section{Results: laboratory study}

A $2 \times 2$ (lighting condition by sampling time) within-subjects, analysis of variance (ANOVA) was performed using melatonin concentrations as the outcome measure. There was a significant main effect of lighting condition $\left(F_{1,10}=17.6 ; P=0.002\right.$; partial $\eta^{2}=0.64$ ) and a significant lighting condition by sampling time interaction $\left(F_{1,10}=14.8 ; P=0.003\right.$; partial $\left.\eta^{2}=0.6\right)$.

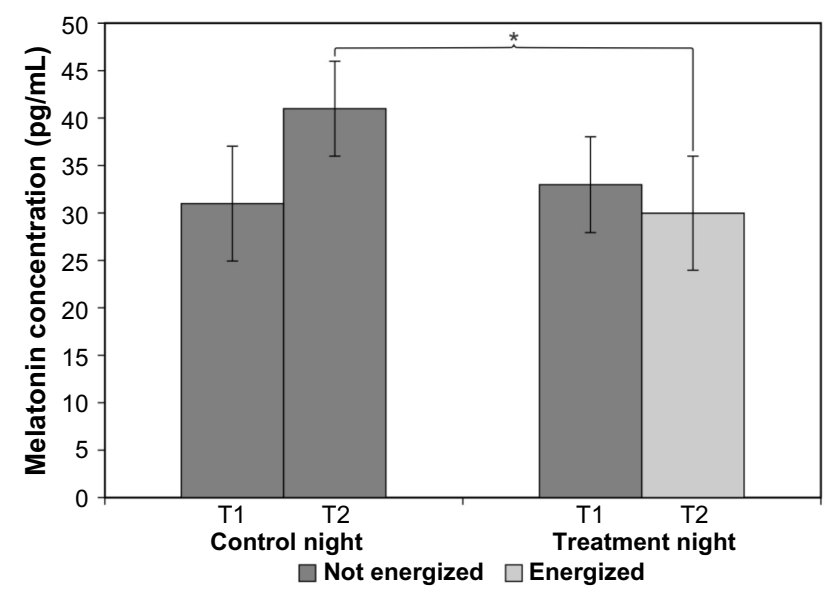

Figure I Mean \pm standard error of the mean of the melatonin concentrations $(\mathrm{pg} / \mathrm{mL})$ at $\mathrm{TI}$ (always in darkness) and T2 (darkness or after I hour exposure to the blue light pulses).

Notes: During the control night, the sleep mask was not energized and during the intervention night, the sleep mask was energized at T2, delivering the blue light pulses. Melatonin concentrations increased from TI to T2 when the sleep mask was not energized and decreased when it was energized. *Statistically significant.
The statistically significant interaction from the ANOVA (Figure 1) is supported by post hoc Student's $t$-tests. Melatonin concentrations for the second sampling period (T2) of the control night (mean \pm standard error of the mean [SEM] = $41 \pm 6 \mathrm{pg} / \mathrm{mL}$ ), when the sleep mask was not energized, were significantly greater $(P<0.05)$ than melatonin concentrations for T2 of the treatment night (mean \pm SEM $=30 \pm 5 \mathrm{pg} / \mathrm{mL}$ ), when the light mask was energized, demonstrating that the light from the sleep mask significantly suppressed melatonin concentrations. Melatonin concentrations at T1, for the control and treatment nights, when samples were always collected in darkness, were not significantly different (mean $\pm \mathrm{SEM}=33 \pm 5$ and $31 \pm 6 \mathrm{pg} / \mathrm{mL}$, respectively). Therefore, melatonin concentrations increased from $\mathrm{T} 1$ to $\mathrm{T} 2$ when the sleep mask was not energized and decreased when it was energized. The mean \pm SEM melatonin suppression was $16.6 \% \pm 8 \%$.

\section{Results: field study}

A one-factor (weeks) ANOVA revealed that DLMO times were significantly different between weeks $\left(F_{1,9}=16.4\right.$; $P=0.003$; partial $\eta^{2}=0.65$ ). DLMO times after intervention were significantly later than at baseline. Table 1 shows the DLMO values at baseline and after the 1-week light intervention for every subject. As shown in Table 1, all but one subject delayed DLMO after the intervention. The mean \pm SEM DLMO phase delay (calculated by subtracting DLMO after intervention from DLMO at baseline) was $-24 \pm 5$ minutes. A two-tailed, one-sample $t$-test revealed that DLMO delay was significantly different than zero $(t=3.96 ; P=0.003)$.

Table I DLMO times (h:min) at baseline and after the light intervention for every subject together with his or her respective phase shifts (baseline time minus intervention time; negative number $=$ phase delay)

\begin{tabular}{llll}
\hline & $\begin{array}{l}\text { Baseline } \\
\text { (h:min) }\end{array}$ & $\begin{array}{l}\text { After intervention } \\
\text { (h:min) }\end{array}$ & $\begin{array}{l}\text { Shift in DLMO } \\
\text { (h:min) }\end{array}$ \\
\hline $20: 03$ & $20: 06$ & -0.03 \\
& $20: 45$ & $21: 06$ & -0.21 \\
$20: 07$ & $20: 34$ & -0.27 \\
& $21: 22$ & $21: 27$ & -0.05 \\
& $20: 18$ & $21: 03$ & -0.45 \\
& $20: 45$ & $21: 30$ & -0.45 \\
& $21: 10$ & $21: 37$ & -0.27 \\
& $21: 46$ & $21: 36$ & 0.10 \\
Average & $19: 15$ & $19: 54$ & -0.39 \\
SEM & $19: 00$ & $19: 37$ & -0.37 \\
\hline
\end{tabular}

Note: Associated average and variance values (mean \pm SEM) are also provided. Abbreviations: DLMO, dim light melatonin onset; SEM, standard error of the mean. 
Figure 2 shows the mean \pm SEM KSS values obtained during the first day and the last day of the intervention week. Two-tailed paired Student's $t$-tests revealed that self-reports of sleepiness at dinner time were significantly lower (ie, subjects were less sleepy) on the last day of the intervention week compared to the first day.

Sleep efficiency during the baseline and intervention weeks and sleep start times on the first day and on the last day of the intervention week were calculated from the actigraph data using the Actiware-Sleep Version 3.4 from Mini Mitter Co, Inc (now Philips Respironics, Pittsburg, PA, USA). The mean \pm SEM sleep efficiency was $82 \% \pm 2 \%$ during the baseline week and $81 \% \pm 2.7 \%$ during the intervention week. Sleep efficiency was not significantly different $(t=0.48$; $P=0.65$ ) between the 2 weeks of the study. Sleep start times on the first day of the intervention week were delayed by 16 minutes compared to sleep start times on the last day of the intervention week, but this difference was not statistically significant $(t=1.9 ; P=0.09)$.

\section{Discussion}

The results of the laboratory study confirm those previously obtained with the sleep mask, ${ }^{20}$ but there was no apparent advantage of doubling the frequency of blue light pulses in terms of melatonin suppression. The mean melatonin suppression observed in the earlier study was $14 \%$, close to the $16.6 \%$ observed in the present study. These results are also consistent with Zeitzer et al, who reported that an even shorter duration of light pulse (60 2-millisecond pulses delivered over 1 hour)

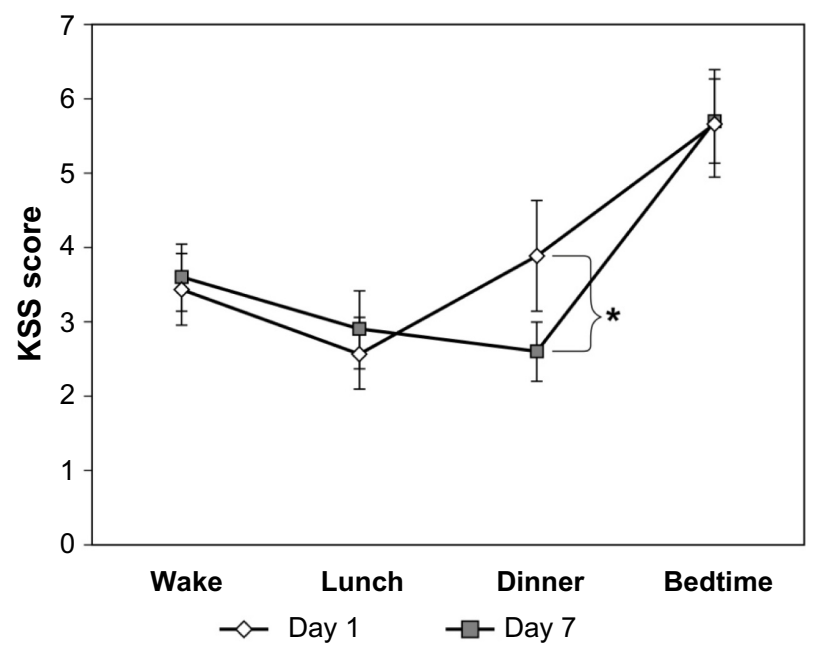

Figure 2 Mean \pm standard error of the mean KSS values collected during the first and last day of the intervention week.

Notes: Subjects were significantly less sleepy at dinner time after I week of the light intervention (day 7) than on the first day of the intervention week (day 1). *Statistically significant.

Abbreviation: KSS, Karolinska Sleepiness Scale. when eyes were open suppressed melatonin by approximately $34 \%$, even though, due to large individual differences, there was no significant difference in melatonin suppression between the flash and the dark control condition. ${ }^{32}$ Rea et al hypothesized, as an extension of their model, ${ }^{18,19}$ that a train of blue light pulses could be effective for reliably stimulating the human circadian system. ${ }^{20}$ This hypothesis was again tested and validated here. It should be clear, however, that the temporal dynamics of the human circadian system are more poorly understood than its spectral and absolute sensitivities. More basic research is needed to elucidate the temporal characteristics of human circadian phototransduction.

The results of the field study also confirm previous laboratory findings showing that a train of blue light pulses can delay circadian phase, ${ }^{20}$ but these are the first to demonstrate the feasibility of wearing a sleep mask at home to deliver light treatments through closed eyelids during sleep. The magnitude of the phase shifting observed after 1 week of wearing the sleep mask at home (approximately 24 minutes) was similar to that observed after 1 day of wearing the same sleep mask in the laboratory (approximately 32 minutes), most likely because subjects in the field study experienced uncontrolled light exposures during the day, especially during the morning hours, which would have counteracted the delaying light from the sleep mask. Future studies should investigate whether pulsing blue light through closed eyelids during sleep in combination with short-wavelength-filtering orange-tinted glasses worn in the morning would result in greater phase delays.

DLMO was significantly delayed following the light intervention. Consistently, the actigraph data showed that, compared to the first night of the intervention week, the average sleep start time on the last night of the intervention week (ie, after 7 days of light treatment) was delayed by 16 minutes. Since the exact clock times of KSS scores were unreported, it is not possible to directly link the lower subjective sleepiness from the KSS scores at dinner time to the later measured DLMO. It seems reasonable to infer, however, that because the objective measures from both DLMO and actigraphy are consistent with the lower KSS scores at dinner time, the light intervention was in fact effective at delaying the subjects' physiology.

We informally asked the subjects about the comfort of wearing the sleep mask during sleep. The overall consensus was that the sleep mask woke subjects up on the first night of light treatment, but that by the third night, they had become used to the mask. Some subjects continued to wake up at the first pulse of light, but would immediately fall back asleep, while others slept soundly through the light pulses. Regardless 
of these initial self-reported sleep interruptions, average sleep efficiency, as measured by the actigraph data, was similar in both, at baseline and during the intervention weeks. Moreover, unpublished polysomnography measurements in our laboratory indicated that subjects $(n=6)$ had a number of micro-arousals during exposure to the pulsing light, but these were not significantly different than those measured during the control night when the sleep mask was not energized. Also from an eye safety perspective, the brief pulses of blue light minimize risk of blue light hazard, ${ }^{33}$ even if people might have inadvertently had their eyelids open during the brief pulses. And from a thermal burn perspective, the demonstrated effectiveness of applying a train of blue light pulses through closed eyelids during sleep makes the development of a sleep mask for home use practical because it avoids the significant heat buildup from continuous LED operation. Given the demonstrated treatment efficacy as well as user compliance and safety, this method of light treatment offers great promise for clinical applications aimed at correcting circadian misalignment. A systematic examination of efficacy and compliance to different forms of light treatment should be the focus of future research.

The present studies do have limitations. The sample sizes were relatively small, so future research, particularly studies conducted in the field, should be repeated with a larger population. Currently, our laboratory is conducting a larger study utilizing the sleep mask to delay DLMO and sleep times in older adults with and without sleep onset insomnia. The subjects who participated in the laboratory study were younger than those in the field study because one of our goals of the laboratory study was to investigate whether doubling the frequency of the light pulses would result in more acute melatonin suppression. We therefore chose to use an age range in the laboratory study that was comparable to the one used in our previous published study. ${ }^{20}$ It is not known whether younger adults would have responded to the light treatment during early sleep like the older subjects. Future studies should investigate how the light treatment during sleep can affect DLMO in different age groups.

In conclusion, the results of the laboratory study confirmed previous results using the sleep mask showing that a train of blue light pulses can reliably suppress nocturnal melatonin;20 however, doubling the frequency of pulses from once per minute to twice per minute did not significantly increase the magnitude of suppression. Understanding the temporal dynamics of the human circadian system is a critical area for future basic research. The results of the field study showed, for the first time, that the train of blue light pulses can be used to delay circadian phase in older adults living at home.
These results have important implications for those who suffer from circadian sleep disorders, such as delayed sleep phase disorder. Importantly, the subjects in the present experiment felt less sleepy at dinner time and, if reliably observed, this would be beneficial to those who suffer from early sleep onset and fall asleep too early in the evening. Future studies should be performed to include those suffering from advanced sleep phase disorder as well as delayed sleep phase disorder. It will be interesting to investigate whether delivery of pulsing blue light in the early morning after predicted $\mathrm{CBT}_{\min }$ can advance the timing of circadian phase and of sleep onset, particularly among teenagers and college students who have delayed sleep onset times and associated shorter sleep durations.

\section{Acknowledgments}

The study was funded by the National Institute on Aging (\# R01AG042602). Rebekah Mullaney, Greg Ward, Andrew Bierman, Sharon Lesage, Erin Ryan, Dennis Hull, and Dennis Guyon of the Lighting Research Center are acknowledged for their technical and editorial support.

\section{Disclosure}

The authors have filed, in conjunction with Philips Lighting, a patent application on the flashing blue light mask. The authors report no other conflicts of interest in this work.

\section{References}

1. Sateia M, Nowell P. Insomnia. Lancet. 2004;364(9449):1959-1973.

2. Leger D, Scheuermaier K, Philip P, Paillard M, Guilleminault C. Evaluation of quality of life in severe and mild insomniacs compared with good sleepers. Psychosom Med. 2001;63(1):49-55.

3. Brassington GS, King AC, Bliwise DL. Sleep problems as a risk factor for falls in a sample of community-dwelling adults aged 64-99 years. J Am Geriatr Soc. 2000;48:1234-1240.

4. Blackwell T, Yaffe K, Ancoli-Israel S, et al. Poor sleep is associated with impaired cognitive function in older women: the SOF study. J Gerontol A Biol Sci Med Sci. 2006;61(4):405-410.

5. Buysse DJ, Reynolds CF, Monk TH, Hoch CC, Yeager AL, Kupfer DJ. Quantification of subjective sleep quality in healthy elderly men and women using the Pittsburgh Sleep Quality Index (PSQI). Sleep. 1991;14(4):331-338.

6. Maggi S, Langlois JA, Minicuci N, et al. Sleep complaints in community-dwelling older persons: prevalence, associated factors, and reported causes. J Am Geriatr Soc. 1998;46(2):161-168.

7. Ford DE, Kamerow DB. Epidemiologic study of sleep disturbances and psychiatric disorders. An opportunity for prevention. JAMA. 1989;262: 1479-1484.

8. Lustberg L, Reynolds CF. Depression and insomnia: questions of cause and effect. Sleep Med Rev. 2000;4(3):253-262.

9. Kupfer DJ. Pathophysiology and management of insomnia during depression. Ann Clin Psychiatry. 1999;11(4):267-276.

10. Sharma M, Palacios-Bois J, Schwartz G, et al. Circadian rhythms of melatonin and cortisol in aging. Biol Psychiatry. 1989;25(3):305-319.

11. Kirisoglu $C$, Guilleminault $C$. Twenty minutes versus forty-five minutes morning bright light treatment on sleep onset insomnia in elderly subjects. J Psychosom Res. 2004;56(5):537-542. 
12. Münch M, Scheuermaier KD, Zhang R, et al. Effects on subjective and objective alertness and sleep in response to evening light exposure in older subjects. Behav Brain Res. 2011;224(2):272-278.

13. Lieverse R, Van Someren EJ, Nielen MM, Uitdehaag BM, Smit JH, Hoogendijk WJ. Bright light treatment in elderly patients with nonseasonal major depressive disorder: a randomized placebocontrolled trial. Arch Gen Psychiatry. 2011;68(1):61-70.

14. Hamilton M. A rating scale for depression. J Neurol Neurosurg Psychiatry. 1960;23(1):56.

15. Suhner AG, Murphy PJ, Campbell SS. Failure of timed bright light exposure to alleviate age-related sleep maintenance insomnia. $J \mathrm{Am}$ Geriatr Soc. 2002;50(4):617-623.

16. Figueiro MG, Rea MS. Preliminary evidence that light through the eyelids can suppress melatonin and phase shift dim light melatonin onset. BMC Res Notes. 2012;5(1):221.

17. Bierman A, Figueiro MG, Rea MS. Measuring and predicting eyelid spectral transmittance. J Biomed Optics. 2011;16(6):067011.

18. Rea MS, Figueiro MG, Bullough JD, Bierman A. A model of phototransduction by the human circadian system. Brain Res Rev. 2005;50(2): 213-228.

19. Rea MS, Figueiro MG, Bierman A, Hamner R. Modelling the spectral sensitivity of the human circadian system. Light Res Technol. 2012;44(4):386-396.

20. Figueiro MG, Bierman A, Rea MS. A train of blue light pulses delivered through closed eyelids suppresses melatonin and phase shifts the human circadian system. Nat Sci Sleep. 2013;5:133-141.

21. World Medical Association. Declaration of Helsinki. JAMA. 2000; 284(23):3043-3045.

22. Roenneberg T, Wirz-Justice A, Merrow M. Life between clocks: daily temporal patterns of human chronotypes. J Biol Rhythms. 2003;18(1): 80-90.
23. Rea MS, Figueiro MG, Bierman A, Bullough JD. Circadian light. J Circadian Rhythms. 2010;8(1):2.

24. Bierman A, Klein TR, Rea MS. The Daysimeter: a device for measuring optical radiation as a stimulus for the human circadian system. Meas Sci Technol. 2005;16:2292-2299.

25. Figueiro MG, Hamner R, Bierman A, Rea MS. Comparisons of three practical field devices used to measure personal light exposures and activity levels. Light Res Technol. 2013;45(4):421-434.

26. Ancoli-Israel S, Cole R, Alessi C, Chambers M, Moorcroft W, Pollak CP. The role of actigraphy in the study of sleep and circadian rhythms. Sleep. 2003;26(3):342-392.

27. Martin SK, Eastman CI. Sleep logs of young adults with self-selected sleep times predict the dim light melatonin onset. Chronobiol Int. 2002;19(4):695-707.

28. Åkerstedt T, Gillberg M. Subjective and objective sleepiness in the active individual. Int J Neurosci. 1990;52(1-2):29-37.

29. Voultsios A, Kennaway DJ, Dawson D. Salivary melatonin as a circadian phase marker: validation and comparison to plasma melatonin. J Biol Rhythms. 1997;12(5):457-466.

30. Smith MR, Revell VL, Eastman CI. Phase advancing the human circadian clock with blue-enriched polychromatic light. Sleep Med. 2009;10(3):287-294.

31. Molina TA, Burgess HJ. Calculating the dim light melatonin onset: the impact of threshold and sampling rate. Chronobiol Int. 2011;28(8): 714-718.

32. Zeitzer JM, Ruby NF, Fisicaro RA, Heller HC. Response of the human circadian system to millisecond flashes of light. PLoS One. 2011;6(7):e22078.

33. Bullough JD. The blue-light hazard: a review. J Illum Eng Soc. 2000;29(2):6-14.
Nature and Science of Sleep

\section{Publish your work in this journal}

Nature and Science of Sleep is an international, peer-reviewed, open access journal covering all aspects of sleep science and sleep medicine, including the neurophysiology and functions of sleep, the genetics of sleep, sleep and society, biological rhythms, dreaming, sleep disorders and therapy, and strategies to optimize healthy sleep. The journal welcomes

\section{Dovepress}

original research, clinical \& epidemiological studies, reviews \& evaluations, case reports and extended reports. The manuscript management system is completely online and includes a very quick and fair peerreview system, which is all easy to use. Visit http://www.dovepress.com/ testimonials.php to read real quotes from published authors. 